\title{
Home Advantage in Judo: Analysis by the Combat Phase, Penalties and the Type of Attack
}

\author{
by \\ Ciro José Brito ${ }^{1}$, Bianca Miarkaํ, Alfonso López Díaz de Durana², David H. Fukuda ${ }^{3}$
}

Previous studies indicate positive home advantage in judo; however, the factors that influence home advantage have yet to be fully explored. This study investigated the potential differences in technical-tactical variables between home and visiting athletes. A total of 1411 video recorded matches were analyzed (123 home, 1288 away) from 36 international judo competitions contested in 2011-12. The matches were analyzed by the following criteria: combat phases (approach, gripping, attack, defense, groundwork and pause), penalties (by the athlete or the opponent) and the types of attacks (with or without a score). Elite judo athletes competing in their home country attacked more frequently using trunk/leg couple techniques $(p<0.011)$ and scored more frequently with arm/leg couple techniques $(p<0.001)$, while visiting judo athletes received fewer scores from penalties $(p<0.001)$ and engaged in more frequent pauses during competition $(p<0.01)$. The results of this study provide an outline of technical-tactical differences that may contribute to home advantage in judo.

Key words: home advantage, martial arts, performance analysis.

\section{Introduction}

An advanced understanding of factors, i.e. home advantage, that could modify the behavior patterns of elite judo athletes and promote changes in physical and psychological preparation for international competition is needed (Franchini et al., 2014). With the recent creation of the world rankings (IJF, 2016), the level of competition has increased while athletes and coaches have used various strategies to benefit from seeding opportunities and secure qualification for the Olympic Games (Ferreira Julio et al., 2013). In 2013, the International Judo Federation limited the participation of athletes, with a maximum of 2 per weight category and 9 per gender for each country in the world championships (Krumer, 2014). In addition to reporting greater odds of athletes winning a medal while competing in their home country, Ferreira Julio et al. (2013) noted that $64 \%$ of the competitions in 2009 occurred in Europe. The competitions with the greatest potential for earning points towards the world-ranking list are designated as Grand Slam and Grand Prix events worth 300 and 200 points, respectively. In 2015, all five Grand Slam (Tokyo, Abu Dhabi, Paris, Tyumen, Baku) and nine Grand Prix (Jeju, Qingdao, Tashkent, Ulaanbaatar, Budapest, Zagreb, Samsun, Tbilisi, Dusseldorf) events were based in Asia and Europe (IJF, 2015). Therefore, any potential home advantage in judo might translate into ranking advantages for Asian and European athletes compared to athletes from other continents. The points earned on the world ranking list and competitive record prior to 2012

\footnotetext{
1 - Physical Education Department, Federal University of Juiz de Fora.

2 - Sports Department. Physical Activity and Sports Faculty. Technical University of Madrid, Madrid, Spain

3 - Institute of Exercise Physiology and Wellness, University Central Florida.
} 
explained about $25 \%$ of the variance in performance at the London Olympics (Franchini and Julio, 2015). This information combined with the analysis of technical-tactical variables from the competitive environment could provide valuable information on how home advantage might affect judo matches.

During judo competition, combat actions and pause phases are highly diversified and the relationships between these variables collectively represent an athlete's system of attack (Miarka et al., 2015). Previous investigations have shown specific factors that influence technical and tactical arrangements in combat sports, including expertise (Calmet and Ahmaidi, 2004; Del Vecchio et al., 2011), the competitive outcome (Chaabène et al., 2014; Escobar-Molina et al., 2014; Miarka et al., 2016), the competitive level (Pozo et al., 2011), weight categories (Courel et al., 2014; Miarka et al., 2015; Tabben et al., 2015), the judging system (Myers et al., 2013) and crowd noise (Myers et al., 2012). Regarding judo competition, age effects have been observed in the time dedicated to the approach, gripping, groundwork and pause phases during tournaments (Miarka et al., 2012, 2014). Despite these differences in time-motion variable analysis, the effects of home advantage on the athlete's system of attack have yet to be observed.

Various reports have identified specific factors, including the approach and grip attempts, gripping patterns (kumi-kata) and effective attacks, that affect judo performance and competitive success (Calmet et al., 2010; Courel et al., 2014; Franchini et al., 2008; Lech et al., 2015). For example, investigation of grip laterality and throwing side preferences has shown that attacks from the same gripping arrangement have greater probability of resulting in a successful score in both male and female athletes (Courel et al., 2014; Kajmovic and Radjo, 2014). Recently, Sterkowicz et al. (2013) noted that, despite no significant differences between the frequencies of scoring techniques in men and women, techniques relying on a force couple were used less frequently (39.6\%) than the techniques utilizing a physical lever (60.5\%). Researchers have also demonstrated that elite female judo athletes receive fewer penalties compared to their male counterparts (Franchini et al., 2008). Winning athletes have shown to attempt more effective attacks than losing athletes after being penalized (EscobarMolina et al., 2014). Consequently, it is recommended that notational analysis in judo should be conducted with consideration for penalties as well as the wide range of interconnected elements within each combat phase, such as the type of approach (Calmet et al., 2010), the type of attacks (Sterkowicz et al., 2013), defensive actions (Boguszewski, 2011) and groundwork attempts (Miarka et al., 2014).

Home advantage is well established in team sports (Gómez et al., 2011), but is underresearched and somewhat controversial in individual sports (Jones, 2013) with the potential for home disadvantage reported in tennis (Gayton et al., 2009). To our knowledge, few studies have investigated home advantage in combat sports, especially in judo (Ferreira Julio et al., 2013; Franchini and Julio, 2015; Krumer, 2014). While male judo athletes win more matches, and both male and female athletes have a greater chance of winning a medal when competing at home (Ferreira Julio et al., 2013), the influence of home advantage on technical and tactical variables has yet to be examined. Therefore, the aim of this study was to investigate home advantage during international judo competition with respect to the phases of combat, penalties and types of attacks. We hypothesized that judo athletes competing at home displayed an advantage in technical-tactical variables compared to visiting athletes.

\section{Material and Methods}

\section{Participants}

The present study considered competitive judo matches contested in 2011-2012 by 154 male and 98 female athletes, representing each of the seven weight classes, who qualified for the 2012 Olympic Games. A randomized selection of 1411 individual combat performances from international competitions was considered, with 123 performances featuring athletes competing in their home country (winning combats $=88$ and $73 \%$ of home country combats; losing combats = 33 and $27 \%$ of home country combats) and 1288 performances featuring athletes competing as visitors to a foreign country (winning combats $=$ 765 and $59 \%$ of foreign country combats; losing combats $=523,41 \%$ of foreign country combats). The present study ensured anonymity and confidentiality by replacing the athletes' personal 
identification. There are no ethical issues in analyzing or interpreting data obtained at public events (Ferreira et al., 2012). In order to guarantee the ecological validity, video recordings documented in publically-available judo databases were utilized as previously described (Miarka et al., 2011). For this purpose, judo matches from 36 international competitions were documented including the Olympic Games, World Championships, World Masters, Grand Slams, Grand Prix's and World Cups, according to the International Judo Federation's 2011-2012 calendar and data (2012). The free computer version of VirtualDub Program 1.8.6(2) was used to fragment and edit images. The local Ethics and Research Committee of the State University of São Paulo previously approved this study.

\section{Study design}

This study addresses the development of a judo combat model through the description and comparison of technical-tactical indicators with previously established criteria (Escobar-Molina et al., 2014; Franchini et al., 2008; Lech et al., 2015; Miarka et al., 2012). The investigation was divided into three stages. First, the combat phases and technical-tactical indicators were identified and incorporated into a conceptual model (EscobarMolina et al., 2014; Franchini et al., 2008; Lech et al., 2015; Miarka et al., 2012). Next, intra and interexpert validation of the model was conducted with a randomized selection of the combat records (Del Vecchio et al., 2011; Miarka et al., 2014). Lastly, differences among time-motion and technical-tactical indicators of the home and visiting athletes were examined.

\section{Protocol of time-motion analysis and intra and inter-expert validation}

The combat phases were examined according to the frequency of occurrence. The protocol followed previous reports, which included the approach (Invernizzi et al., 2015), gripping (Miarka et al., 2014), attack (with and without a score) (Lech et al., 2015), defense (taesabaki) (Calmet, 2007), groundwork combat (Miarka et al., 2014), pause and penalties (by the athlete or by the opponent) (Escobar-Molina et al., 2014). The approach phase was defined by the time between the signal from the referee announcing the beginning of combat ("hajime") and the execution of the grip (kumi-kata). In this period, athletes do not have any physical contact between them, based on the protocol used in the analysis of combat periods in previous reports (Calmet et al., 2010; Miarka et al., 2012). The gripping phase (kumi-kata) was defined by the time between the accomplishment of the grip (kumi-kata) and the lack of contact on the opponent judogi, based on the protocol used in the analysis of the combat time frame in preceding reports (Calmet et al., 2010; Courel et al., 2014; Miarka et al., 2012). The groundwork (ne-waza) phase was defined as the period where one or both fighters executed groundwork techniques, according to the rules, following a previously described protocol (Miarka et al., 2011). The pause phase was defined as the period between the signal for combat interruption ("matte") and the signal for the restart of the combat (command voice "hajime"). The present study did not observe "sono-mama" and "yoshi" commands (halt of the fight and restart), which would also have been computed as recovery if they had had occurred, following a previously described protocol (Miarka et al., 2012).

The attack phase of combat was characterized by the specific biomechanical principles utilized, which were identified by the type of the force couple applied or the length and point of application of the moment arm, as outlined by Sterkowicz et al. (2013). Throwing techniques that employed a force couple were designated as using: an arm couple, an arm/leg couple or a trunk/leg couple, while techniques described by the moment arm were designated as minimal length (applied at the opponent's waist), medium length (applied at the opponent's knee), variable length (below the opponent's waist) or maximal length (applied at the opponent's foot/ankle) (Sacripanti, 2012; Sterkowicz et al., 2013). The defense phase of combat was categorized by the manner in which the defending athlete changed his/her body position and orientation, right or left (tae-sabaki), in response to an attack and by the use of a counter-attack (Boguszewski, 2011). During the pause phase, penalties were counted according to scores received by the athlete or awarded to the opponent as determined by the referee officiating the match (Escobar-Molina et al., 2014; Franchini et al., 2013).

Results from Mann-Whitney $U$ tests ( $p \leq$ 0.05 ) showed no differences among intra and inter- 
expert measurement of the protocol variables. The correlation between measurements obtained for each model was verified by means of the Cohen Kappa coefficient (Lawrence et al., 2014), with an inter intra-agreement range between 0.45 and 1.00, with the classification of "Almost perfect" for Groundwork, Pause, Defense and Penalties; "Strong" for Approach, Trunk/leg couple and Maximal lever, and; "Moderate" for Gripping and Attack variables.

\section{Statistical Analysis}

A logistic regression model was used to verify the probability that an athlete would increase his/her chances of winning when competing in his/her home country. Descriptive results were presented as the odds ratio (OR) at a confidence interval of 95\% (CI95\%; lower limit: LL; upper limit: UL) and a significance level $(p)$. Technical-tactical frequency data are presented as means and standard deviations and Chi-square tests were conducted in order to compare groups. The level of significance of $p \leq 0.05$ was used. All analyses were conducted using SPSS 20.0 for Windows.

\section{Results}

Statistical analysis showed the likelihood of winning was greater for home athletes as compared to away athletes $\left[\chi^{2}=7.209, p=0.007\right.$, OR $(\mathrm{LL} ; \mathrm{UL})=0.582(0.387 ; 0.874)]$. Table 1 presents the results analyzed for the phases of combat and the penalties incurred. The frequencies indicated that visiting judo athletes paused more frequently during competition $(p<$ $0.01)$ and received fewer scores from penalties than home athletes $(p<0.001)$.

Table 2 presents the frequency of results analyzed by the attack type. The home athletes attacked more frequently with arm/leg couple techniques that resulted in scores $(p<0.001)$ and with trunk/leg couple techniques without scoring $(p<0.011)$.

Table 1

Frequency of combat phases and penalties during elite judo competition.

\begin{tabular}{|c|c|c|c|c|c|c|c|c|c|}
\hline \multirow[b]{2}{*}{ Phase } & \multicolumn{3}{|c|}{ Home Athletes } & \multicolumn{3}{|c|}{ Visiting Athletes } & \multicolumn{3}{|c|}{ Statistics } \\
\hline & Mean \pm SD & Min & Max & Mean \pm SD & Min & Max & $\chi^{2}$ & $\mathrm{df}$ & $p$ \\
\hline Approach & $19.9 \pm 13.7$ & 2 & 80 & $20.1 \pm 12.0$ & 1 & 75 & 56.733 & 58 & .522 \\
\hline Gripping & $18.0 \pm 11.4$ & 0 & 53 & $19.0 \pm 11.6$ & 1 & 67 & 68.315 & 58 & .167 \\
\hline Attack & $8.0 \pm 6.9$ & 0 & 39 & $8.3 \pm 6.4$ & 0 & 49 & 35.393 & 34 & .402 \\
\hline Defense & $4.4 \pm 4.1$ & 0 & 22 & $4.8 \pm 4.1$ & 0 & 24 & 26.756 & 24 & .316 \\
\hline Groundwork & $5.2 \pm 4.0$ & 0 & 19 & $6.3 \pm 4.8$ & 0 & 27 & 14.944 & 25 & .943 \\
\hline Pause* & $9.1 \pm 7.0$ & 0 & 38 & $9.9 \pm 6.4$ & 0 & 35 & 53.330 & 32 & .01 \\
\hline \multicolumn{10}{|l|}{ Penalties } \\
\hline $\begin{array}{l}\text { Score received by } \\
\text { the athlete* }\end{array}$ & $0.2 \pm 0.5$ & 0 & 2 & $0.1 \pm 0.4$ & 0 & 3 & 15.890 & 3 & .001 \\
\hline $\begin{array}{l}\text { Score awarded to } \\
\text { the opponent }\end{array}$ & $0.2 \pm 0.5$ & 0 & 3 & $0.2 \pm 0.5$ & 0 & 3 & 2.571 & 4 & .632 \\
\hline \multicolumn{10}{|c|}{$\begin{array}{c}\text { SD - Standard Deviation, Min - minimum, Max - Maximum df-Degrees of } \\
\text { freedom. }{ }^{*} p<.05 \text { Home vs. Visiting athletes. }\end{array}$} \\
\hline
\end{tabular}


Table 2

Frequency of the attack type (with and without a score) during elite judo competition.

\begin{tabular}{|c|c|c|c|c|c|c|c|c|c|}
\hline \multirow{2}{*}{ Type of attack } & \multicolumn{3}{|c|}{ Home Athletes } & \multicolumn{3}{|c|}{ Visiting Athletes } & \multicolumn{3}{|c|}{ Statistics } \\
\hline & Mean \pm SD & Min & Max & Mean \pm SD & Min & Max & $\chi^{2}$ & df & $P$ \\
\hline Arm couple & $0.2 \pm 0.6$ & 0 & 4 & $0.2 \pm 0.6$ & 0 & 5 & 1.312 & 5 & .934 \\
\hline Arm/leg couple & $3.6 \pm 4.9$ & 0 & 33 & $3.2 \pm 4.0$ & 0 & 28 & 25.689 & 25 & .424 \\
\hline $\begin{array}{l}\text { Arm/leg couple } \\
\text { with score* }\end{array}$ & $0.2 \pm 0.5$ & 0 & 3 & $0.1 \pm 0.3$ & 0 & 2 & 30.019 & 3 & .0001 \\
\hline Minimum lever & $0.2 \pm 0.6$ & 0 & 4 & $0.4 \pm 1.0$ & 0 & 16 & 4.760 & 8 & .783 \\
\hline Trunk/leg couple* & $1.5 \pm 2.7$ & 0 & 17 & $1.6 \pm 2.6$ & 0 & 25 & 37.115 & 20 & .011 \\
\hline $\begin{array}{l}\text { Trunk/leg couple } \\
\text { with score }\end{array}$ & $0.1 \pm 0.3$ & 0 & 2 & $0.1 \pm 0.3$ & 0 & 2 & .065 & 2 & .968 \\
\hline Variable lever & $1.2 \pm 2.0$ & 0 & 14 & $1.7 \pm 2.4$ & 0 & 15 & 12.545 & 15 & .637 \\
\hline $\begin{array}{l}\text { Variable lever with } \\
\text { score }\end{array}$ & $0.03 \pm 0.2$ & 0 & 1 & $0.1 \pm 0.4$ & 0 & 3 & 3.180 & 3 & .365 \\
\hline Maximum lever & $0.9 \pm 1.5$ & 0 & 7 & $0.9 \pm 1.5$ & 0 & 10 & 5.205 & 10 & .877 \\
\hline $\begin{array}{l}\text { Maximum lever } \\
\text { with score }\end{array}$ & $0.1 \pm 0.3$ & 0 & 2 & $0.1 \pm 0.3$ & 0 & 4 & .162 & 3 & .983 \\
\hline
\end{tabular}

SD - Standard Deviation, Min - minimum, Max - Maximum df-Degrees of freedom. ${ }^{*} p<.05$ Home vs. Visiting athletes.

\section{Discussion}

With an uneven distribution of international judo competitions, home advantage may provide some assistance for athlete's competing in their home country to gain additional points towards the IJF rankings (Ferreira Julio et al., 2013). The results of this study identify technical-tactical variables, including the number of pauses, penalties incurred and the types of attacks that differ between home and visiting judo athletes during competition. Gaining an understanding of these variables may assist in the development of strategy by national teams, as under the current rules, with few exceptions, each country can take only one athlete to the World Championships and Olympic Games (Krumer, 2014).

Crowd noise, motivation and judges have previously been identified as potential factors influencing competitive success through home advantage (Pollard, 2008). Despite the phenomenon being observed in combat sports, few studies have attempted to explain the factors that promote the advantage. Myers et al. (2012) observed the influence of crowd noise on the judges' decision in favor of the home athlete during Muay Thai fights. Similarly, in boxing, Balmer et al. (2005) noted that home advantage was likely related to subjective judging criteria. This hypothesis might not apply strictly to judo because referees can consult the video replay in case of doubt. In fact, the video replay appeal is of particular importance as judo referees may be influenced by conformism (Boen et al., 2013).

Our results showed that home athletes benefited more frequently from penalties resulting in scores. This finding may be related to 
the greater volume of non-scoring leg techniques (ashi-waza) by the home athletes which could have resulted in non-combativity penalties being incurred by the visiting athletes. In line with these results, Escobar-Molina et al. (2014) revealed that combativeness was crucial in avoiding penalties and influencing opponents to commit them. Indeed, our data also showed that visiting athletes tended to engage in a significantly greater number of pauses when compared to home athletes, which could lead to non-combativity penalties. Forcing an opponent to commit penalties is an increasingly used tactic in modern judo (Escobar-Molina et al., 2014). Franchini et al. (2013) observed that recent rule changes increased the number of penalties, while reducing the techniques resulting in scores, during the European championships.

In the present study, judo athletes competing at home attempted more frequently techniques utilizing force couple techniques (arm/leg couple and trunk/leg couple). Analyzing the results of the 2012 Olympic Games, Sterkowicz et al. (2013) observed that while athletes preferred physical lever techniques, force couple techniques were more effective during competition. Finally, the arm/leg couple techniques were the only types of attacks in which a difference in scoring potential was found between home and visiting athletes. Similar to previous studies that analyzed home advantage in judo at the international level, our results point to a potential benefit for the athletes competing in their home countries. Despite the three existing papers having adopted different methodologies and distinct analysis points (Ferreira Julio et al., 2013; Franchini and Julio, 2015; Krumer, 2014), the findings appear to be in agreement. Our study provides an outline of the technical-tactical differences that may contribute to home advantage in judo. Thus, coaches and athletes can develop competitive strategies for events contested at home and abroad. Future studies should investigate other factors, including the presence of the crowd noise and psychological motivation, which may influence this phenomenon. In conclusion, elite judo athletes competing in their home country attack more frequently using trunk/leg couple techniques and score more frequently with arm/leg couple techniques, while visiting judo athletes receive fewer scores from penalties and engage in more frequent pauses during competition.

\section{Acknowledgements}

To CAPES. Ciro Brito would like to thank the Brazilian Scholarship Program Ciência sem Fronteiras (Grant \#234243/2014-7). Funding received for this work: Fundação CAPES - Ministério da Educação do Brasil

\section{References}

Balmer NJ, Nevill AM, Lane AM. Do judges enhance home advantage in European championship boxing? J Sports Sci, 2005; 23: 409-416

Boen F, Ginis P, Smits T. Judges in judo conform to the referee because of the reactive feedback system. Eur J Sport Sci, 2013; 13: 599-604

Boguszewski D. Defensive actions of world top judoists. J Hum Kinet, 2011; 27: 111-122

Calmet M. Developing ecological research in judo. Percept Mot Skills, 2007; 105: 646-648

Calmet M, Ahmaidi S. Survey of advantages obtained by judoka in competition by level of practice. Percept Mot Skills, 2004; 99: 284-290

Calmet M, Miarka B, Franchini E. Modeling of grasps in judo contests. Int J Perform Anal Sport, 2010; 10: 229-240

Chaabène H, Franchini E, Miarka B, Selmi MA, Mkaouer B, Chamari K. Time-motion analysis and physiological responses to karate official combat sessions: is there a difference between winners and defeated karatekas. Int J Sports Physiol Perform, 2014; 9: 302-308

Courel J, Franchini E, Femia P, Stankovic N, Escobar-Molina R. Effects of kumi-kata grip laterality and throwing side on attack effectiveness and combat result in elite judo athletes. Int J Perform Anal Sport, 2014; 14: 138-147 
Del Vecchio FB, Hirata SM, Franchini E. A review of time-motion analysis and combat development in mixed martial arts matches at regional level tournaments. Percept Mot Skills, 2011; 112: 639-648

Escobar-Molina R, Courel J, Franchini E, Femia P, Stankovic N. The impact of penalties on subsequent attack effectiveness and combat outcome among high elite judo competitors. Int J Perform Anal Sport, 2014; 14: 946-954

Ferreira Julio U, Panissa VLG, Miarka B, Takito MY, Franchini E. Home advantage in judo: A study of the world ranking list. J Sports Sci, 2013; 31: 212-218

Franchini E, Julio UF. The Judo World Ranking List and the Performances in the 2012 London Olympics. Asian J Sports Med, 2015; 6: e24045

Franchini E, Takito MY, Calmet M. European Judo Championships: impact of the new rule changes on points and penalties. Int J Perform Anal Sport, 2013; 13: 474-479

Franchini E, Brito CJ, Fukuda DH, Artioli GG. The physiology of judo-specific training modalities. J Strength Cond Res, 2014; 28: 1474-1481

Franchini E, Sterkowicz S, Meira CM, Gomes FR, Tani G. Technical variation in a sample of high level judo players. Percept Mot Skills, 2008; 106: 859-869

Gayton W, Steinroeder W, Bonnica C, Loinon A. An investigation of home disadvantage in Davis Cup tennis. Percept Mot Skills, 2009; 109: 382-386

Gómez MA, Pollard R, Luis-Pascual J-C. Comparison of the home advantage in nine different professional team sports in Spain 1. Percep Mot Skills, 2011; 113: 150-156

IJF. International Judo Federation Calendar 2015. 2015. Available at: http://www.intjudo.eu/News/cikk3268; accessed on 01.06 .2016

IJF. Internartional Judo Federation World Ranking List. 2016. Available at: http://www.intjudo.eu/foRankingir; accessed on 25.01.2016

Invernizzi PL, Longo S, Bizzi M, Benedini S, Merati G, Bosio A. Interpretation and Perception of Two Different Kumite Fighting Intensities through an Integrated Approach Training in International Level Karatekas: An Exploratory Study. Percept Mot Skills, 2015; 121: 333-349

Jones MB. The home advantage in individual sports: An augmented review. Psychol Sport Exerc, 2013; 14: 397-404

Kajmovic H, Radjo I. Techniques Efficiency Index in Judo between Male and Female Judoka during Bosnia and Herzegovina Senior Phase Championships. Int J Perform Anal Sport, 2014; 14: 620-634

Krumer A. On Winning Probabilities, Weight Categories, and Home Advantage in Professional Judo. J Sports Econ, 2014; Ahead of Print: 1527002514560576

Lawrence DW, Hutchison MG, Cusimano MD, Singh T, Li L. Interrater agreement of an observational tool to code knockouts and technical knockouts in mixed martial arts. Clin J Sport Med, 2014; 24: 397-402

Lech G, Chwala W, Ambrozy T, Sterkowicz S. Muscle torque and its relation to technique, tactics, sports level and age group in judo contestants. J Hum Kinet, 2015; 45: 167-175

Miarka B, Vecchio FBD, Camey S, Amtmann J. Comparisons technical-tactical and time-motion analysis of mixed martial arts by outcomes. J Strength Cond Res, 2016; 30: 1975-1984

Miarka B, Hayashida CR, Julio UF, Calmet M, Franchini E. Objectivity of FRAMI-software for judo match analysis. Int J Perform Anal Sport, 2011; 11: 254-266

Miarka B, Branco BH, Vecchio FB, Camey S, Franchini E. Development and validation of a time-motion judo combat model based on the Markovian Processes. Int J Perform Anal Sport, 2015; 15: 315-331

Miarka B, Coswig VS, Vecchio FB, Brito CJ, Amtmann J. Comparisons of Time-motion Analysis of Mixed Martial Arts Rounds by Weight Divisions. Int J Perform Anal Sport, 2015; 15: 1189-1201

Miarka B, Panissa VL, Julio UF, Del Vecchio FB, Calmet M, Franchini E. A comparison of time-motion performance between age groups in judo matches. J Sports Sci, 2012; 30: 899-905 
Miarka B, Cury R, Julianetti R, Battazza R, Julio UF, Calmet M, Franchini E. A comparison of time-motion and technical-tactical variables between age groups of female judo matches. J Sports Sci, 2014; 32: 1529-1538

Myers T, Nevill A, Al-Nakeeb Y. The influence of crowd noise upon judging decisions in Muay Thai. Adv Phys Edu, 2012; 2: 148-152

Myers T, Nevill A, Al-Nakeeb Y. A comparison of the effect of two different judging systems on the technique selection of Muay Thai competitors. J Hum Sport Exerc, 2013; 8: 761-777

Pollard R. Home advantage in football: A current review of an unsolved puzzle. Open Sports Sci J, 2008; 1: 12-14

Pozo J, Bastien G, Dierick F. Execution time, kinetics, and kinematics of the mae-geri kick: comparison of national and international standard karate athletes. J Sports Sci, 2011; 29: 1553-1561

Sacripanti A. Biomechanical revision of the principles of Dr. Jigoro Kano's judo kodokan. Med Dello Sport, 2012; 65: 265-281

Sterkowicz S, Sacripanti A, Sterkowicz-Przybycien K. Techniques frequently used during London Olympic judo tournaments: a biomechanical approach. Arch Budo, 2013; 1: 51-58

Tabben M, Coquart J, Chaabène H, Franchini E, Ghoul N, Tourny C. Time-motion, tactical and technical analysis in top-level karatekas according to gender, match outcome and weight categories. J Sports Sci, 2015; 33: 841-849

\section{Corresponding author:}

\section{Ciro José Brito}

Faculdade de Educacao Fisica e Desporto - FAEFID

José Lourenço Kelmer st. - Martelos, Juiz de Fora - MG, Brazil. ZIP. 36036-330

Phone: +55(32) 2102-3911,

FAX +55(32) 2102-3989

E-mail: cirojbrito@gmail.com 